\title{
Regenerative Medicine in Endodontics: Time to Look Beyond the Root Canal Treatment
}

\section{Preetham Kodimoole*}

Department of Dental Sciences, Narayana Dental College, Nellore, India

"Corresponding author: Preetham kodimoole, Department of Dental Sciences, Narayana Dental College, Nellore, India, Tel: + 09901189035; E-mail: kpree99@gmail.com

Received date: December 02, 2016; Accepted date: January 06, 2017; Published date: January 12, 2017

Copyright: @ 2017 Kodimoole P. This is an open-access article distributed under the terms of the Creative Commons Attribution License, which permits unrestricted use, distribution, and reproduction in any medium, provided the original author and source are credited.

Keywords: Dentistry; Human population; Regenerative medicine; Endodontics; Crown cutting; Endodontics treatment; Periodontitis; Root canal; Dentin complex; Tooth decay

\section{Short Communication}

Our dentistry has quite evolved from the past. Now as we are in the 21st century the priorities and the attitude of the general human population towards the dental treatment has evolved differently upto a certain extent. Generally the general population have a gut feeling about Endodontics is they generally know as a Root Canal Specialists. In the early 2001 and 2004 ceratin dental scientists and molecular biologists came up with this bizarre idea of introduction of regenerative medicine in Endodontics [1].

\section{What is Regenerative Medicine Then?}

Regenerative medicine is dealing with the replacement, engineering and regenerating the human cells and the tissues, organs to restore or establish normal function. How this method was introduced to the Endodontics was an Intresting topic for me. Traditionally speaking the time is up for the dentistry to pave the way for the new ideas and technological innovations. The old classical way of dental treatments may not hold too long as there is still a lot more scope to manipulate and to operate the procedure for the more of foolproof success. The biggest disadvantage in the RCT procedure is the tooth is biologically dead, a huge amount of tooth structure is lost, with subsequent Crown cutting and Crown replacement. Now what if the the necrosed tooth pulp is restored with normal blood supply to form a growth? In normal procedures it seems to be imposssible but in the Regenerative Endodontic procedure it is posible [2]. Here the regenerative procedure stands out differently from the root canal procedure. The dentists can quite think differently instead of treating a class 2 cavity with a RCT procedure. Do a class 2 cavity require a RCT procedure? It is all together a different topic. It is certainly an individual's choice (Figure 1).

\section{Where Do the Dentists Lack?}

The goal of regenerative endodontics treatment is to save immature teeth with pulpal necrosis, to describe disease of the pulp, the material found inside of the teeth. When the pulpal necrosis is not treated, the affected tooth die and must be removed. With regenerative endodontics, the dental pulp can be rehabilitated, so that the teeth can continue to develop normally.

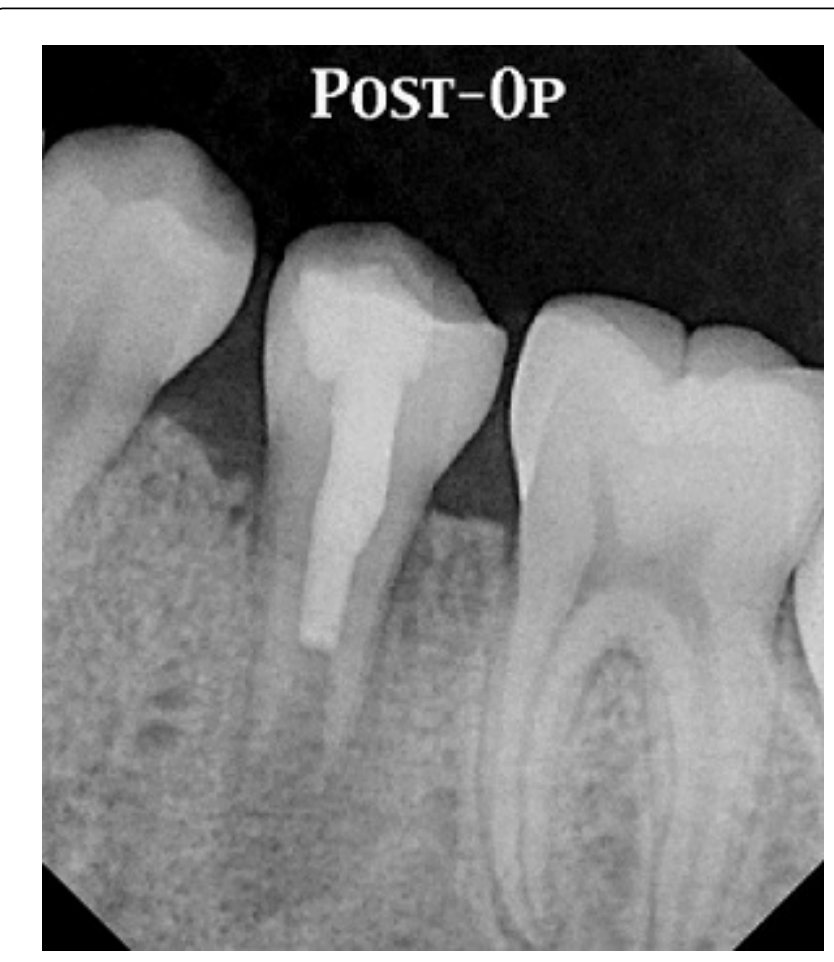

Figure 1: Introduction of regenerative medicine in Endodontics.

Success rate for regeneretive endodontic procedures: It has shown that healing process of the apical periodontitis, total development of the root apex and thickness of the root canal wall of immature teeth with pulpal necrosiscan occur after regenerative endodontic procedures. At present the success rate is about 70 percent. It will increase as more and more funding is done by the dental foundations on this research. The cost for this procedure is quite expensive as compared to the RCT, the procedure innovation is on the initial stage. This may give a boost to the dentistry that deserves.

\section{Conclusion}

Regenerative procedures are different from traditional endodontic techniques in that they only use chemical means of cleaning the root canal system(s), chemical means of cleaning the root canal system, placement of scaffolds, and the traditional means of chemicomechanical disinfecting of the root canal system. Regenerative endodontic treatments hold true that a functional pulp dentin complex can be regenerated for the patients. While a complete overhaul of the 
Citation: Kodimoole P (2017) Regenerative Medicine in Endodontics: Time to Look Beyond the Root Canal Treatment. Dentistry 7: 407. doi: 10.4172/2161-1122.1000407

Page 2 of 2

regenerative endodontic procedures are done on the immature teeth researchers are now experimenting whether these types of treatment can be done in fully developed teeth. It is an exciting time in the field of endodontics. Who knows what may happen? This procedure has the potential to be added as a superspecialisation course after masters degree in endodontics.

\section{References}

1. Li X, Masanori N (2014) From regenerative dentistry to regenerative medicine: progress, challenges, and potential applications of oral stem cells. Stem Cells Cloning 7: 89-99.

2. Ramta B, Aditya J (2015) Current overview on dental stem cells applications in regenerative dentistry. J Nat Sci Biol Med 6: 29-34. 